\title{
Frenkel-Exciton-Polaritons in Organic Microcavities
}

\author{
Hashem Zoubi and G. C. La Rocca \\ Scuola Normale Superiore and INFM, Piazza dei Cavalieri 7, 56126 Pisa, Italy
}

Received on 4 April, 2005

\begin{abstract}
We study organic microcavities in the strong coupling regime. The cavity exciton-polariton dispersion relations and quantum states are derived in using a microscopic theory. We consider the two cases of anisotropic organic crystals with one and two molecules per unit cell. In general, the cavity exciton-polaritons are a coherent superposition of both cavity mode polarizations and of both Davydov exciton branches. The obtained polarization mixing is in contrast to the case of typical inorganic semiconductor cavities in which no mixing between the TM and TE polarizations occurs. By applying the quasi-mode approximation, we derive the transmission, reflection, and absorption coefficients for high quality organic cavities.
\end{abstract}

Keywords: Frenkel-exciton-polaritons; Organic microcavities; Strong coupling regime

The interest in organic and inorganic microcavities is in their ability to control the coupling between photons and electronic excitations. In the strong coupling regime, where the photon-exciton interaction is larger than the exciton and photon damping rates, the cavity photons and the excitons are coherently coupled to produce the cavity exciton-polaritons. The polariton dispersion relation splits into branches which are separated by the Rabi splitting frequency. In typical quantumwell semiconductor microcavities, the coupling between the Wannier-Mott excitons and the cavity photons yields Rabi splitting values of the order of $10 \mathrm{meV}$ [1]. The large oscillator strength of the organic materials makes the use of organic microcavities more attractive. The strong coupling between the Frenkel excitons in organic materials and the cavity photons results in a Rabi splitting which is easily an order of magnitude larger than that of inorganic microcavities [2]. In particular, a strong coupling regime has been observed in an organic microcavity containing $J$ aggregates of cyanine dye where the Rabi splitting is between $80 \mathrm{meV}$ and $300 \mathrm{meV}$, at room temperature [3]. Such materials are disordered, but the case of crystalline organic media is also of great current interest [4].

We study the Frenkel-exciton-polaritons of an organic microcavity in the strong coupling regime and their linear optics spectra on the basis of the microscopic theory. M. Litinskaia et al. [5] have investigated such system in the framework of the macroscopic approach based on the use of the dielectric tensor. D. Balagurov and one of us [6] have calculated the linear optics spectra of such system using a phenomenological uniaxial dielectric tensor and a $(4 \times 4)$ transfer matrix formalism.

First, we represent the cavity photons. The optical confinement in the microcavity is provided by two parallel mirrors at a distance of the order of an optical wavelength. Initially, the mirrors are assumed to be perfect and no dissipation mechanism is included. The electromagnetic field is free in the cavity plane with in-plane wave vector $\mathbf{q}$, and is confined in the perpendicular $z$ direction with wave vector $q_{z}$, which has the values $q_{z}=m \pi / L$, where $(m=1,2,3, \cdots)$. The cavity-mode dispersion is given by

$$
\omega_{\mathbf{q} m}=\frac{c}{\sqrt{\varepsilon}} \sqrt{q^{2}+\left(\frac{m \pi}{L}\right)^{2}}, q=|\mathbf{q}|,
$$

where $\varepsilon$ is the background dielectric constant of the medium between the mirrors. For each in-plane wave vector $\mathbf{q}$ there are two possible polarizations: $(s)$-modes with transverse electric field, which has only $T$ component. $(p)$-modes with transverse magnetic field, which has $L$ and $Z$ components. Only one relevant cavity-mode at a time in the $z$ direction is considered, the one which is close to resonance with the organic slab excitons.

At the center of the microcavity is placed a slab of organic crystal of width small compared to an optical wavelength, made of $N$ mono-layers parallel to the microcavity planes. The organic crystal is composed of molecules which are all chemically identical and have inversion symmetry. The excitons are free in the slab plane with in-plane wave vector $\mathbf{k}$, and are confined in the $z$ direction with discrete wave vector $k_{z}$, which takes the values $k_{z} a=\pi l /(N+1)$, with $(l=1,2, \cdots, N)$, where $a$ is the distance between each two neighbor mono-layers. In the slab only interactions between nearest neighbor mono-layers are assumed.

We derive the Frenkel-exciton dispersion relation. In the case of crystals with one molecule per unit cell, the exciton dispersion is

$$
\hbar \omega^{e x}\left(\mathbf{k}, k_{z}\right)=\hbar \omega_{0}+J(\mathbf{k}, 0)+J\left(\mathbf{k}, k_{z}\right),
$$

where $\omega_{0}$ is the molecule transition frequency in the crystal. The exciton dynamical matrix $J(\mathbf{k}, 0)$ is for interactions between molecules in the same mono-layer, and $J\left(\mathbf{k}, k_{z}\right)$ is for interactions between molecules from two nearest neighbor mono-layers.

The dynamical matrices are calculated for the case of a cubic crystal with lattice constant $a$, where each molecule has the same transition dipole moment $\vec{\mu}=\left(\vec{\mu}_{\|}, \mu_{z}\right)$. The interaction between the crystal molecules is given by the dipoledipole interaction. In the long wave limit, that is $k a \ll 1$, where $k=|\mathbf{k}|$, the exciton dynamical matrices are given by

$$
J\left(\mathbf{k}, k_{z}\right)=\frac{4 \pi}{3 a^{3}} \cos \left(k_{z} a\right) e^{-a k}(k a)\left\{3\left(\vec{\mu}_{\|} \cdot \hat{k}\right)^{2}-\mu_{z}^{2}\right\},
$$

and

$$
J(\mathbf{k}, 0)=\frac{F}{a^{3}}\left\{2 \mu_{z}^{2}-\mu_{\|}^{2}\right\}
$$


where $F \approx 4.5$, and $\hat{k}=\mathbf{k} / k$. Weak anisotropy effects, of the order of $k a$, are obtained. The difference between the exciton dispersion for the $z$ and the in-plane dipole moment cases, at $k=0$, results in a polarization splitting, which was obtained also in inorganic semiconductor thin layers [7].

In organic slab with two molecules per unit cell, the molecules have the transition dipole moments $\vec{\mu}_{1}$ and $\vec{\mu}_{2}$, they differ only for the orientation, where $\left|\vec{\mu}_{1}\right|=\left|\vec{\mu}_{2}\right|$. We define the two orthogonal transition dipole moments $\vec{\mu}_{ \pm}=\frac{\vec{\mu}_{1} \pm \vec{\mu}_{2}}{\sqrt{2}}$. Here, the exciton dispersion splits into two Davydov branches [8]. In the case of in-plane transition dipole moments, each Davydov branch is related to one of the two orthogonal transition dipole moments. While, in the case of general dipole moments with $z$ components, due to the perpendicular broken symmetry, each Davydov exciton branch is related to both orthogonal dipole moments.

Cavity polaritons are a coherent superposition of excitons and photons of both polarizations $(s)$ and $(p)$. This fact is in contrast to the in-organic semiconductor cavities where no polarization mixing exist [7]. As dictated by in-plane translational symmetry, the coupling is between excitons and photons with the same in-plane wave vector. For the coupling the dipole approximation is used. For the excitons only one dominant mode in the $z$ direction is assumed, the lowest energy mode which has no nodes, $(l=1)$. The $Z$ component of the $(p)$ polarized long wave cavity photon is weakly coupled with the excitons, with coupling of the order of $k / Q$, where $Q=\sqrt{k^{2}+(\pi / L)^{2}}$. We study polaritons in organic cavities in the two case of one and two molecules per unit cell.

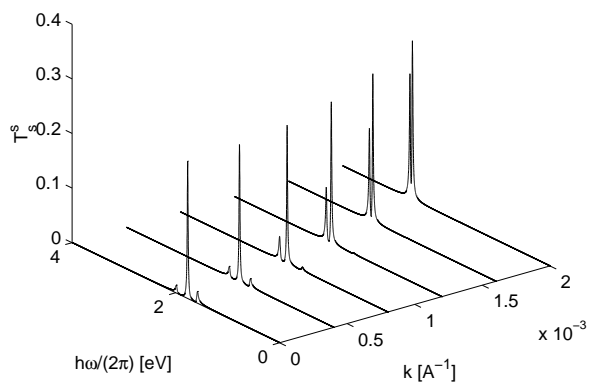

Fig. 1. The $(s)$ polarized field transmission $T_{s}^{(s)}$ vs. wave vector $k$.

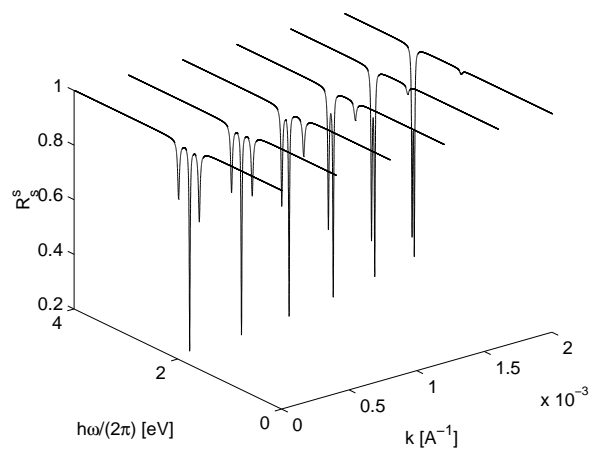

Fig. 2. The $(s)$ polarized field reflection $R_{s}^{(s)}$ vs. wave vector $k$.

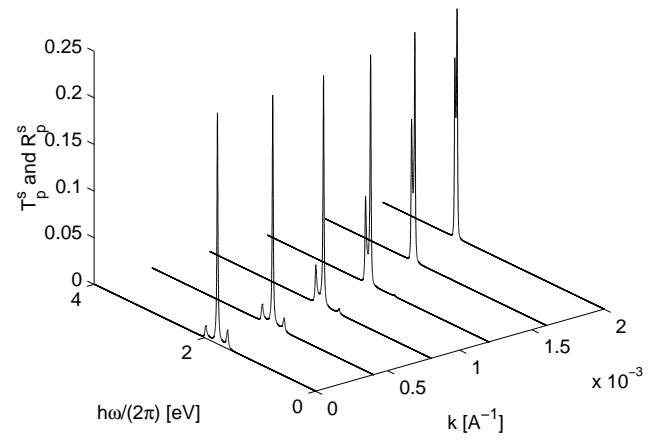

Fig. 3. The $(p)$ polarized field transmission and reflection, $T_{p}^{(s)}$ and $R_{p}^{(s)}$, vs. wave vector $k$.

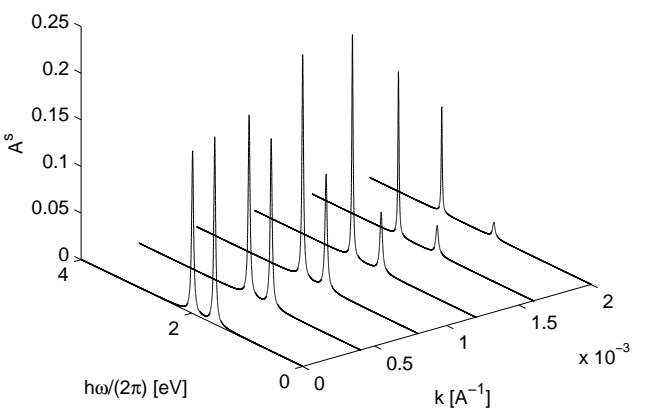

Fig. 4. The absorption $A^{(s)}$ vs. wave vector $k$.

In crystalline organic cavity with one molecule per unit cell, three polariton branches are obtained. Due to the existence of a polarization direction where the excitons and the cavitymodes are decoupled, one is a pure photonic branch. The active mode in the $z$ direction is chosen to be the first one with $(m=1)$, and the molecule dipole moment is $\vec{\mu}=\left(\mu_{x}, 0, \mu_{z}\right)$.

The upper and lower polariton branch dispersions are

$$
\begin{aligned}
& \Omega_{ \pm}(\mathbf{k})=\frac{\omega_{\mathbf{k}}^{e x}+\omega_{\mathbf{k}}^{c a v}}{2} \\
& \pm \sqrt{\left(\frac{\omega_{\mathbf{k}}^{e x}-\omega_{\mathbf{k}}^{c a v}}{2}\right)^{2}+W^{2}(\mathbf{k})\left(1-\frac{k^{2}}{Q^{2}} \cos ^{2} \phi\right)},
\end{aligned}
$$

where $W(\mathbf{k})=\mu_{x} \sqrt{\frac{4 \pi \hbar \omega_{\mathbf{k}}^{c a v}}{L a^{2} \varepsilon}}$, for a single mono-layer slab $(N=$ $1)$, and where $\phi$ is the angle between the in-plane wave vector $\mathbf{k}$ and the $\hat{\mathbf{x}}$ axis. The anisotropy effect in the dispersions is of the order of $(k / Q)^{2}$. In the limit of long waves, that is $Q \gg k$, the dispersion relations are similar to that of isotropic materials.

In order to calculate the linear optical spectra of the microcavity, the quasi-mode approach is used to couple the cavity polaritons to the external photons, assuming non-ideal cavity mirrors [9]. The quasi-mode model is applicable only for a cavity with high quality mirrors, where the cavity photon damping rate, $\gamma$, is related to the mirror reflectivity $R$ by 
$\gamma \propto(1-R)$. The absorption in the cavity medium is included phenomenologically by the non-radiative decay of the excitons, with the exciton damping rate $\Gamma$.

For the case of $(s)$ polarized external input field, the $(s)$ polarized field transmission and reflection, $T_{s}^{s}$ and $R_{s}^{s}$, and the $(p)$ polarized field transmission and reflection, $T_{p}^{s}$ and $R_{p}^{s}$, and the absorption, $A^{s}=1-T_{s}^{(s)}-T_{p}^{(s)}-R_{s}^{(s)}-R_{p}^{(s)}$, are derived.

In figures $(1-4)$, the spectra are plotted for an organic cavity with one molecule per unit cell, for different in-plane wave vectors. Three peaks are seen in corresponding with the three polariton branches. Although the input field is $(s)$ polarized, the anisotropic organic material gives rise also to $(p)$ polarized transmitted and reflected fields, which indicates the polarization mixing. We used typical parameters for organics: the exciton energy, in the limit of small wave vector, is $\hbar \omega^{e x}=1.8 \mathrm{eV}$, the dielectric constant is $\varepsilon=4$, the distance between the cavity mirrors is $L=1750 \AA$, and the excitoncavity mode coupling parameter is $\hbar W=0.2 \mathrm{eV}$. The cavity mirror damping rate is $\hbar \gamma=0.01 \mathrm{eV}$, and the exciton damping rate is $\hbar \Gamma=0.03 \mathrm{eV}$. We choose the direction with $\phi=\pi / 4$.

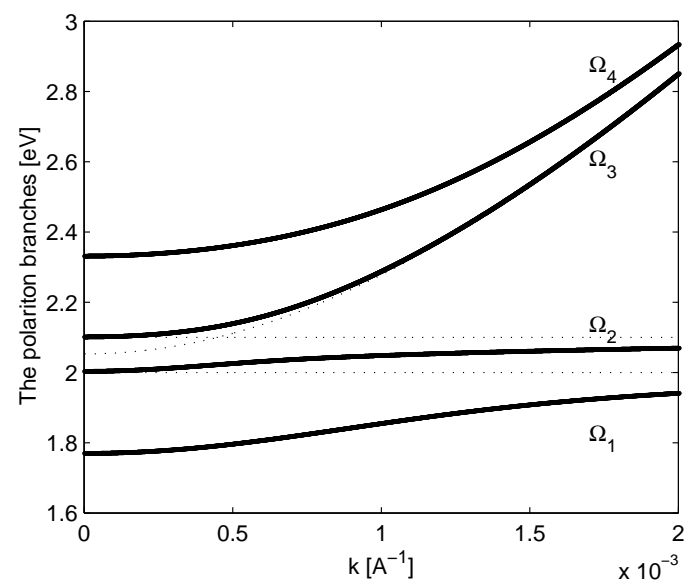

Fig. 5. The four cavity polariton energies vs. in-plane wave vector $k$.

In crystalline organic cavity with two molecule per unit cell, four polariton branches are obtained. In the case where the transition dipole moments are in the slab plane, and in the limit of long waves, the exciton-photon coupling does not mix the two Davydov exciton branches, while the material anisotropy results in a polarization mixing.

In the case of molecule dipole moments with $z$ components, in general the polaritons are a coherent superposition of both Davydov exciton branches and both cavity photon polarizations. The four polariton dispersions, $\Omega_{r}(k)$, are plotted in figure (5). The exciton Davydov branches $(a)$ and $(b)$ weights, $\left|\alpha_{\mathbf{k} r}\right|^{2}$ and $\left|\beta_{\mathbf{k} r}\right|^{2}$, and the cavity photon polarizations $(s)$ and $(p)$ weights, $\left|\gamma_{\mathbf{k} r}\right|^{2}$ and $\left|\delta_{\mathbf{k} r}\right|^{2}$, in the $\Omega_{1}$ and $\Omega_{4}$ polariton branches, are plotted in figures (6) and (7), respectively. A significant mixing between the two Davydov exciton branches appears. The two exciton Davydov energies are chosen to be $\hbar \omega_{a}^{e x}=2 \mathrm{eV}$ and $\hbar \omega_{b}^{e x}=2.1 \mathrm{eV}$. For the cavity we use the dielectric constant $\varepsilon=4$, and the distance between the mirrors is $L=1510 \AA$. The exciton-photon coupling parameters are of the order of the Davydov splitting, that is about $W \sim 0.1 \mathrm{eV}$.

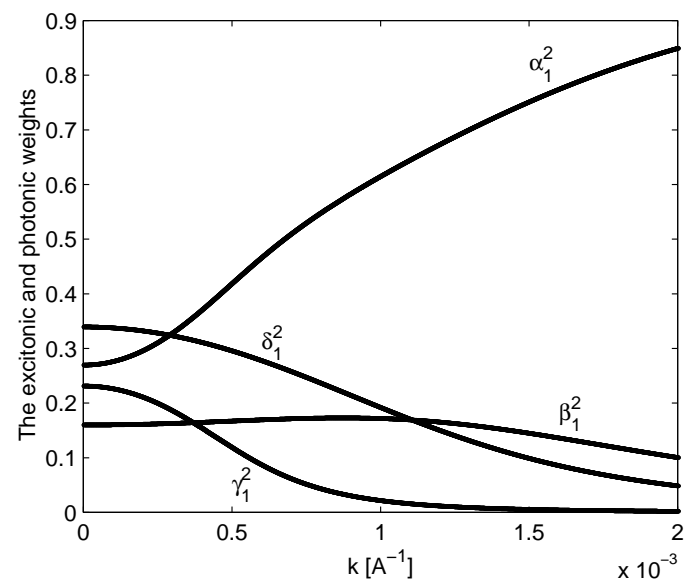

Fig. 6. The excitonic and photonic weights for the first polariton branch.

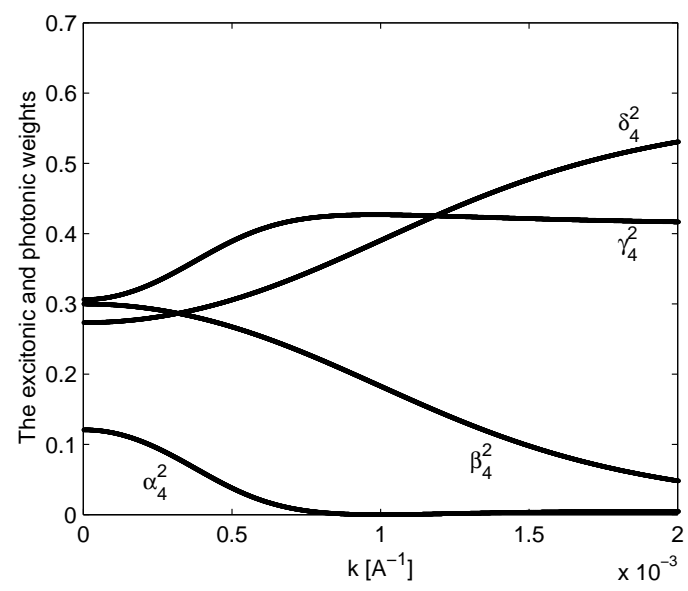

Fig. 7. The excitonic and photonic weights for the fourth polariton branch.

\section{Acknowledgments}

We gratefully acknowledge the support of the European Commission via the $5^{\text {th }}$ Framework Research Training Network HPRN-CT-2002-00315 'The physics of hybrid organicinorganic heterostructures for photonics and telecommunications' (HYTEC).
[1] C. Weisbuch, M. Nishioka, A. Ishikawa, and Y. Arakawa, Phys. Rev. Lett., 69, 3314 (1992).
[2] V. Agranovich, H. Benisty, and C. Weisbuch, Solid State Com- 
mun., 102, 631 (1997).

[3] D. G. Lidzey, D. D. C. Bradley, M. S. Skolnik, T. Virgili, S. Walker, and D. M. Whittaker, Nature 395, 53 (1998).

[4] S. Forrest, Chemical Reviews 97, 1793 (1997).

[5] M. Litinskaya, P. Reineker, and V. M. Agranovich, Phys. Stat. Sol. (a), 201, 646 (2004).

[6] D. B. Balagurov, and G. C. La Rocca, Phys. Stat. Sol. (c), 1, 518
(2004).

[7] L. C. Andreani, and F. Bassani, Phys. Rev. B, 41, 7536 (1990).

[8] A. S. Davydov, Theory of Molecular Excitons, (Plenum, New York, 1971).

[9] V. Savona, C. Piermarocchi, A. Quattropani, P. Schwendimann, and F. Tassone, Phase Transitions, 68, 169 (1999). 